\title{
An Agent-based Approach to Health Care Management
}

\author{
Jun Huang ${ }^{1}$, N. R. Jennings ${ }^{2}$ and John Fox ${ }^{3}$ \\ 1. Dept. of Computing, University of Central Lancashire, Preston PR1 2HE, UK. \\ 2. Dept. of Electronic Engineering, Queen Mary \& Westfield College, Mile \\ End Road, London E1 4NS, UK. \\ 3. Advanced Computation Laboratory, Imperial Cancer Research Fund, \\ 61 Lincoln's Inn Fields, London WC2A 3PX, UK.
}

Abbreviated title: Agent-based health care management

Complete Mailing Address for correspondence:

Dr. Nick Jennings

Dept. of Electronic Engineering

Queen Mary \& Westfield College

Mile End Road.

London E1 4NS

UK 


\begin{abstract}
The provision of medical care typically involves a number of individuals, located in a number of different institutions, whose decisions and actions need to be coordinated if the care is to be effective and efficient. To facilitate this decision making and to ensure the coordination process runs smoothly, the use of software support is becoming increasingly widespread. To this end, this paper describes an agent-based system which was developed to help manage the care process in real world settings. The agents themselves are implemented using a layered architecture, called AADCare, which combines a number of AI and agent techniques: a symbolic decision procedure for decision making with incomplete and conflicting information, a concept of accountability for task allocation, the notions of commitments and conventions for managing coherent cooperation, and a set of communication primitives for interagent interaction. The utility of this approach is demonstrated through the development of an application prototype for the clinical process of cancer treatment.
\end{abstract}




\section{Introduction}

Artificial Intelligence and knowledge based systems are assuming an increasingly important role in medicine for assisting clinical staff in making decisions under uncertainty (eg diagnosis decisions, therapy and test selection, and drug prescribing). Furthermore, many medical procedures now involve several individuals, in a number of specialist institutions (or departments), whose decisions and actions need to be coordinated if the care is to be effective and efficient (Pritchard, 1992; Reeves et al., 1993; Renaud-Salis et al., 1992). For example, a general practitioner (GP) may suspect that his patient has breast cancer. However, as he neither has the knowledge nor the resources to confirm this hypothesis, he must refer the patient to a hospital specialist who can make a firm diagnosis. Having confirmed the presence of breast cancer, the specialist must devise a care programme for treating the patient - this typically involves the hospital, the patient's GP, and a home care organisation jointly executing a series of interrelated tasks. In addition to this inter-organisation coordination, there is also a need to ensure that the activities within an organisation are effectively and coherently managed. In a hospital, for instance, care typically involves execution of interrelated tasks by doctors, nurses, pharmacy, laboratories, and resource management departments.

To provide the appropriate software support for such coordinated health care management it was decided to adopt an agent-based approach. This decision was based on three main observations about the medical care management domain (given below) and the properties of autonomy, social ability, reactivity and proactiveness which are normally associated with intelligent agents (Wooldridge and Jennings, 1995). The first relevant domain property is the fact that there is a significant physical distribution of information, 
problem-solving capabilities, resources, and responsibilities which need to be brought together in a consistent and coherent fashion by the distributed 'agents' who jointly execute a care programme (here agent is defined as an integrated entity involving a computer system and its user). Secondly, the combination of the aforementioned decentralisation and the high cost of obtaining a comprehensive (complete) overview means that decisions often have to be made with incomplete information (eg diagnosis may be proposed without exhaustive laboratory investigation). Finally, as the environment is dynamic and unpredictable the problem solvers need to exhibit intelligent goal-oriented behaviour yet still be responsive to changes in their circumstances - plans to achieve particular goals need to be devised and whilst these plans are being executed they need to be continuously monitored (and perhaps refined) in the light of changes in information and problem solving state.

Given these domain properties and previous experience with medical care management systems, the essential features of an agent-based system for this application area can be defined. Firstly, the agents need explicit communication management procedures (dealing with both syntax and semantics) so that the sender and receiver of a message have a common understanding of its meaning and purpose (in non-automated systems, human to human messages were often misinterpreted during extensive interactions because of ambiguities in the communication structures). Secondly, appropriate mechanisms and structures are needed to ensure that tasks are delegated to the most appropriate agents (previously tasks were allocated to the wrong agents and thus delays in the delivery of care occurred - a serious concern as time is such a critical factor in care administration). Thirdly, the agents require a decision making mechanism which is able to reason with contradictory and incomplete information (previously the popularly used decision 
methods, especially those based on probabilistic theory, could not tolerate conflicting or incomplete information). Finally, to ensure coherent care in spite of the dynamic and unpredictable environment the agents need to specify and adopt an explicit set of procedures for monitoring their goals and plans (previously no explicit procedures existed and changes in goals and care plans were managed largely in an ad-hoc and ineffective manner).

The remainder of this paper is structured in the following manner: section 2 presents a real-world clinical scenario of distributed medical care which is used throughout the remainder of the paper to illustrate the key agent concepts. Section 3 describes the agent architecture, called AADCare, which is based on a three-layer knowledge organisation (domain layer, inference layer and control layer) and is informed by work on The Oxford System of Medicine (Fox et al., 1990) and the KADS model of expertise (Hickman et al., 1989). This section deals, in turn, with each of the key agent features that were identified above. Finally, section 4 compares AADCare with related work.

\section{A clinical scenario of distributed care*}

When an oncologist in a cancer hospital has to treat a patient's breast cancer, the first decision he has to make concerns the treatment plan which will be adopted. To assist him in making this decision, the oncologist consults one of his decision support systems. This system has a built-in decision procedure which is able to deal with incomplete or intuitively inconsistent information, such as evidence in favour of a choice and evidence

\footnotetext{
* This scenario is based on an actual clinical case provided by Fondation Bergonie of Bordeaux, France [(Renaud-Salis et al., 1992). All of the interactions described herein have been implemented using AADCare agents.
} 
against the choice (see section 3.2 for more details). Having weighted the pros and cons of using various treatment options, the system recommends the use of a particular chemotherapy protocol called 'CT1 protocol'. The oncologist authorises use of this protocol and requests the computer system to support him in carrying out the treatment.

The CT1 protocol consists of a number of treatment stages, one of which, stage 2, is shown in Figure 1. According to the protocol, stage 2 is decomposed into a sequence of three subtasks: 'admit patient to hospital', 'administer drugs and monitor patient' and 'discharge patient'. The support system recommends that the oncologist carries out the first task because it knows that he is formally responsible for the admission of his patients. This recommendation is endorsed by the oncologist and consequently he takes on the role of managing and actually performing the activity. On the recommendation of his support system, the oncologist then decomposes 'admit patient to hospital' into two parallel subtasks: 'allocate bed' and 'obtain patient consent'. The machine recommends, and the oncologist accepts, that 'allocate bed' should be performed by the hospital's resource management department and 'obtain patient consent' should be carried out by the oncologist. With respect to the former subtask, the oncologist sends an electronic request to the resource department to see whether they are willing to take on the responsibility for performing it. Assuming they are and that the task is successfully completed, the patient will be allocated a bed. Once a bed is available and the patient agrees to be admitted to the hospital, the support system recommends that the task 'administer drugs and monitor patient' is allocated to a hospital nurse. Assuming she accepts, the protocol dictates that the task should be decomposed into the sequential subtasks of 'obtain drug', 'administer drug' and 'observe patient' - all of which the nurse takes responsibility for. She may subsequently decompose the 'observe patient' subtask still further: for example, into 
'measure body temperature', 'take blood samples', and 'analyse blood samples' and this decomposition may well involve generating a request to a laboratory to test patient indicators, such as white blood cell count. However for the sake of simplicity, this level of decomposition is not be described here. Finally, the machine recommends that the third subtask of stage 2, 'discharge patient', and its two subtasks, 'Instruct Patient' and 'Inform GP' should be carried out by the oncologist.

\section{$<$ FIGURE 1>}

In this scenario, information is transferred according to the following pattern: the resource management department must inform the oncologist about the outcome of 'allocate bed' (i.e. either that a bed has been allocated as requested or that no bed is available for the requested date) and the nurse has to inform the oncologist of the results of 'administer drugs and monitor patient' (e.g. drug has been administered and all patient indicators are normal). Accompanying this information exchange is a concomitant flow of control, in terms of commitments and expectations (Jennings, 1992), between the agents: the oncologist expects the resource management department to perform the activity 'allocate bed' once it has agreed to, similarly he expects the nurse to perform the 'administer drugs and monitor patient' task on time once she has consented to execute it.

\section{AADCare: An agent architecture for distributed medical care}

The AADCare agent architecture comprises multiple layers of knowledge, a working memory, a communications manager and a human-computer interface (see figure 2) (Huang et al., 1995). To be successful in this domain, the agent needs to exhibit both deliberative behaviour (eg plan selection, task decomposition, and task allocation) and reactive behaviour (eg respond in a timely manner to the arrival of new data, to changes in 
existing data, and to varying agent commitments). Within the proposed architecture the deliberative behaviour is achieved by the incorporation of decision rules for plan selection, task management rules for task decomposition and allocation, and cooperation rules for formulating commitments. Reactive behaviour is achieved by the control layer which responds to changes in the working memory (e.g. the arrival of new task results, goals, or messages or changes in existing data, goals, agent commitments or task states).

\section{$<$ FIGURE 2>}

The three layers of knowledge which form the key part of the AADCare architecture are as follows:

- Domain knowledge - includes, for example: a knowledge base covering specific medical domains such as breast cancer, a knowledge base of clinical management plans (known as clinical protocols (Gordon et al., 1993)), a database of patient records, and a database of resource availability.

- Inference knowledge - in the form of generic, declarative inference rules which specify inference relations between domain knowledge, existing patient information, and possible new data. Inference rules represent the core of the agent architecture and are subdivided into those for decision making under uncertainty (section 3.2), those for task management (section 3.3), and those for managing agent cooperation (section 3.4).

- Control knowledge - applies the inference knowledge to the domain knowledge in order to generate new inferences whenever new data is added to the working memory. Logically, this layer is a meta-level which controls the execution of inference rules and domain facts. 
In more detail, the domain knowledge base simply states information and facts about the domain. It says nothing about how the knowledge is to be used. For example, it states that the second stage of the CT1 protocol for treating breast cancer contains three subtasks: 'admit patient to hospital', 'administer drugs and monitor patient' and 'discharge patient':

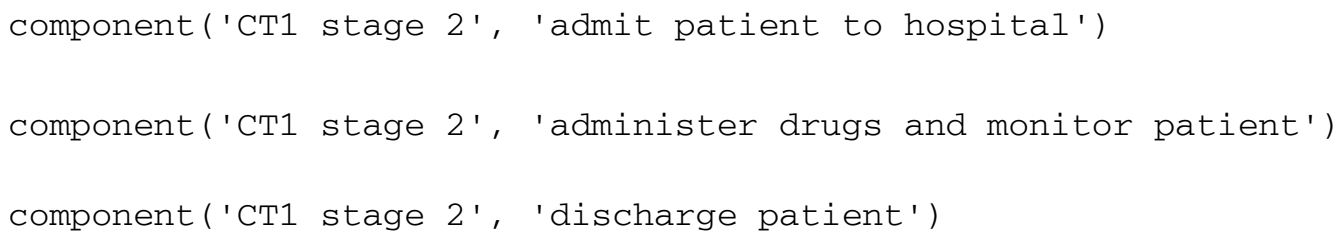

The inference knowledge base contains rules (implemented as declarative schemas) that specify the inference relations between domain-level knowledge and possible new information. For example, the following inference schema specifies that the state of a task becomes 'started' once the state of one of its subtasks becomes 'started':

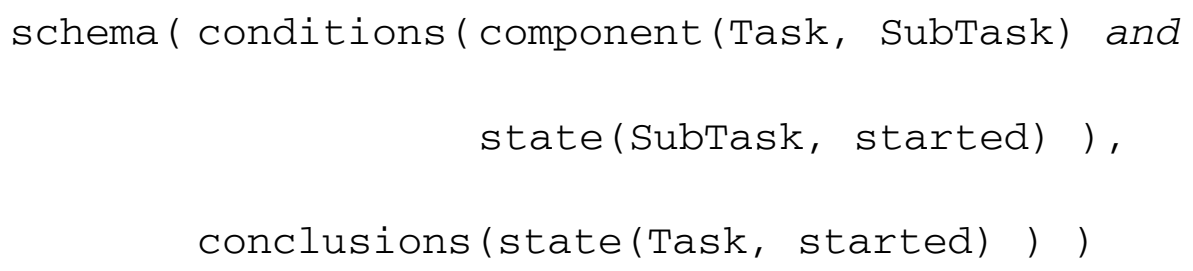

In the context of CT1 protocol, the above schema implies that the task 'CT1 stage 2' should become 'started' when one of its subtasks (e.g. 'admit patient to hospital') becomes 'started' (see section 3.3 for more details of the management of task state transitions).

However, it is only at the control level that the actual execution of the inference rules is carried out and new data is added into the working memory: 
If schema(Conditions, Conclusions) and

\section{al1_true (Conditions)}

then

add (Conclusions)

For example, within the context of CT1 protocol, once the data state('admit patient to hospital', started) is asserted to the working memory, the above control rule applies the given inference schema and domain knowledge to add a new piece of data into the working memory: state ('CT1 stage 2', started).

Bringing all of this together, a sample working session of an oncologist agent is as follows. The oncologist firstly specifies an initial goal of finding an appropriate protocol for treating a particular patient's breast cancer. The goal triggers the control layer to apply the decision rules (section 3.2), medical domain knowledge and patient case data to arrive at a decision (i.e. a suggested treatment protocol called 'CT1 protocol') and associated arguments. The oncologist endorses that decision and requests the machine to assist him in managing the execution of the protocol. This will, in turn, trigger the control layer to apply the task management rules (Section 3.3) to decompose the protocol into constituent tasks, propose task allocations to appropriate agents, and generate the corresponding communication primitives. Once the oncologist accepts the proposal, the communications manager will convert the primitives into complete messages (section 3.1) and send them to the specified agents. Once the chosen agents inform the oncologist of their acceptance of the task requests they and the oncologist enter into a dynamic, cooperating agent community. Agent commitments are generated and monitored through triggering the control layer to apply cooperation rules (section 3.4). Cooperation among the agents 
continues until the entire protocol is in a terminable state (e.g. completed or abandoned). In the meantime, the oncologist may enter into another agent community and accept task requests from other agents.

There are two main reasons for adopting this functional and logical separation of domain, inference and control knowledge. Firstly, it simplifies the representation, reuse and maintenance of knowledge. Inference knowledge for decision making, task management and cooperation can be represented independently of medical domains and can therefore be reused; control knowledge is represented independently of the inference knowledge and so the same control rules can be applied to the three different groups of inference rules. Furthermore, modifications to domain knowledge can be made independently of inference and control knowledge. The second main reason for such a separation is that it provides a convenient basis for knowledge elicitation: domain knowledge can be acquired and modified independently of inference and control knowledge.

AADCare's working memory stores temporary data generated by the control layer, the user, or the communications manager. Examples of the types of information which need to be stored include: goals to be achieved, control states of tasks that are currently active, results of completed tasks, incoming and outgoing messages, and current commitments. Its function is similar to that of a blackboard, on to which new information (or any change which triggers reactions by the control layer) can be added.

The communications manager composes the messages to be sent to the other agents from the primitives produced by firing task management or cooperation rules (see sections 3.3 and 3.4 for respective examples). It also converts messages which arrive from other 
agents into primitives that may be used by the cooperation manager. More details of this module are given in section 3.1.

The human computer interface defines a scheme for interaction between the support system and its user. The approach is as follows: the computer can perform various functions (i.e. decision making, task management, communication and cooperation) but may not act autonomously on all of these capabilities. In general, the computer informs the user of the results of its inferences and the user must then endorse or authorise them before they can be communicated to external agents. For example, the system may recommend to the oncologist that he asks a particular nurse to perform the drugs administration sub-task, however the oncologist may have a personal preference for another nurse and may, therefore, be unwilling to make such a referral. In this case, the system will not send an electronic request to the original nurse, but will instead offer the oncologist an alternative solution.

\subsection{Communication Management}

After an extensive analysis of the interactions which can occur in cooperative care organisations, a set of communication primitives, based on speech act theory (Searle, 1969), have been defined (Table 1). Each primitive has a type (illocutionary force) and a content (propositional content), as well as a certain effect on the receiver (perlocutionary force). Having a well-defined set of primitives is important because it means that the ambiguity in message interchange is substantially reduced - each primitive has a clear meaning and must be responded to in a predictable way. There has been similar work on communication primitives elsewhere (e.g. in the Contract Net Protocol (Smith, 1980), the message perceptor in OFFICE (Winograd and Flores, 1986), IMAGINE's cooperative 
primitives (Lux et al., 1993), and the AGENT0 programming language (Shoham, 1993)), however none of these systems offered an appropriate set for the domain of distributed health care management.

\section{$<$ TABLE $1>$}

The primitives request, accept, reject and alter are used during the allocation of tasks and the formulation of agent commitments. Using the example from section 2 , the oncologist may allocate the task 'administer drugs and monitor patient' to a nurse by requesting her to perform it during a period of ten days starting on the following day. The nurse may accept the task exactly as specified by the oncologist, or she may reject it because she is too busy during the next few days (insufficient resources to honour the commitment). Alternatively, the nurse may indicate that she cannot start the task the following day, but she could start it two days later (alter).

A suggest act may be the result of a query - for instance, suggesting treatment protocol CT1 after being asked how to treat breast cancer. Inform usually follows an accepted request to perform a certain task and is mainly used to disseminate results. Inform may also accompany a request to provide relevant information for the contractor. The fact that cancel is included as a primitive type is because in certain circumstances agents may modify their commitments (as discussed in section 3.4.2). Finally, all messages must be acknowledged.

Knowledge about the semantics of the different types of primitives is incorporated in the task management and cooperation rules, which can dynamically generate message primitives when executed by the control layer. The communications manager then converts these primitives into complete, structured messages using a communication 
protocol that defines the syntax of interagent messages (Huang et al., 1994). Note that the '*' superscript denotes repeated entries and that PRIMITIVE_CONTENT is as defined in

\section{Table 1:}

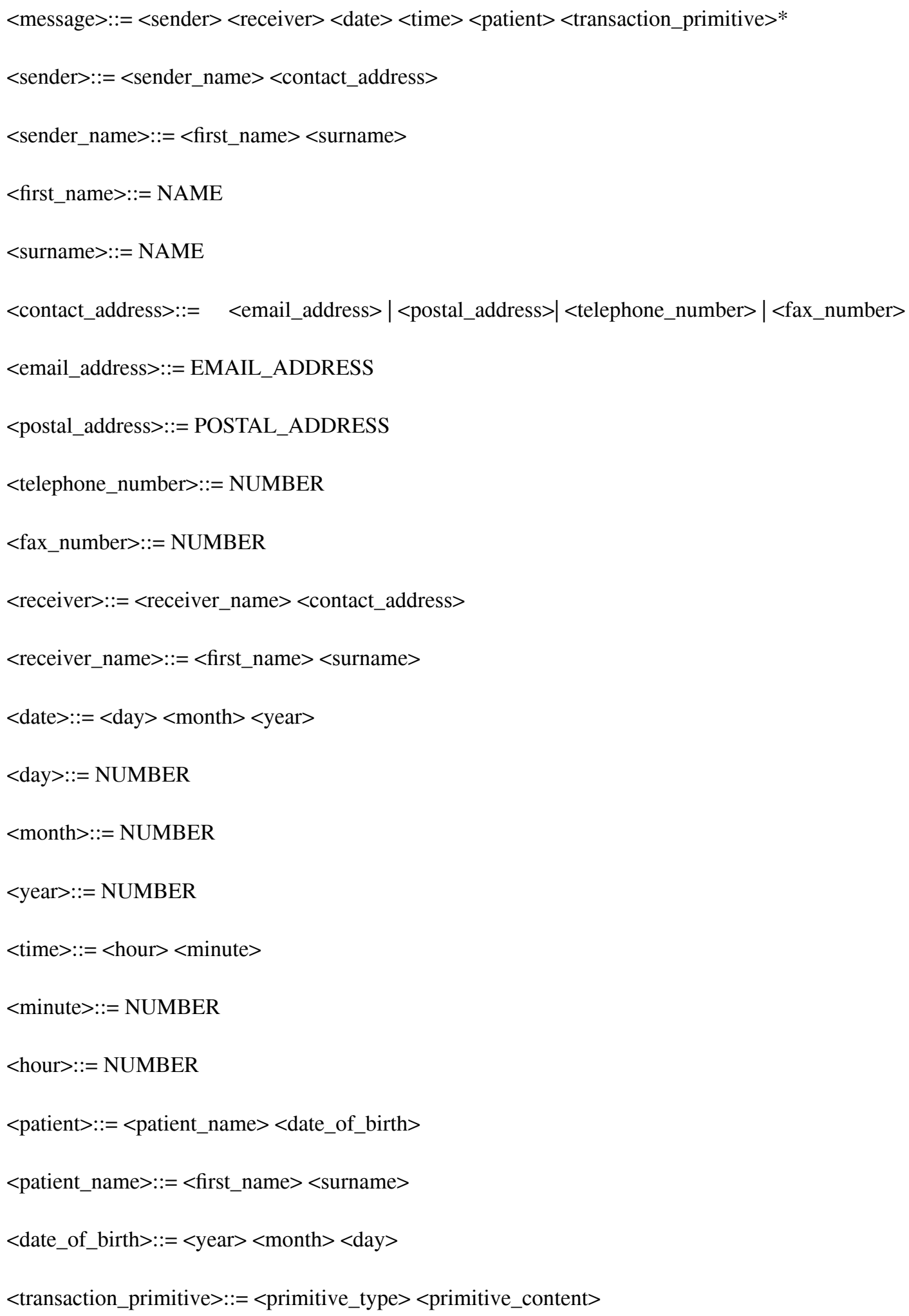


$<$ primitive_type $>::=$

REQUEST | ACCEPT | REJECT | ALTER | PROPOSE | INFORM |

QUERY | CANCEL | ACKNOWLEDGE

$<$ primitive_content $>::=$ PRIMITIVE_CONTENT

By means of an illustration, an agent Jean-Louis Penn may receive the following message from another agent Tony Burg, which includes an urgent request to treat Mary Taylor's breast cancer, as well as some patient data (the most recently-measured tumour size and location) that is thought to be relevant:

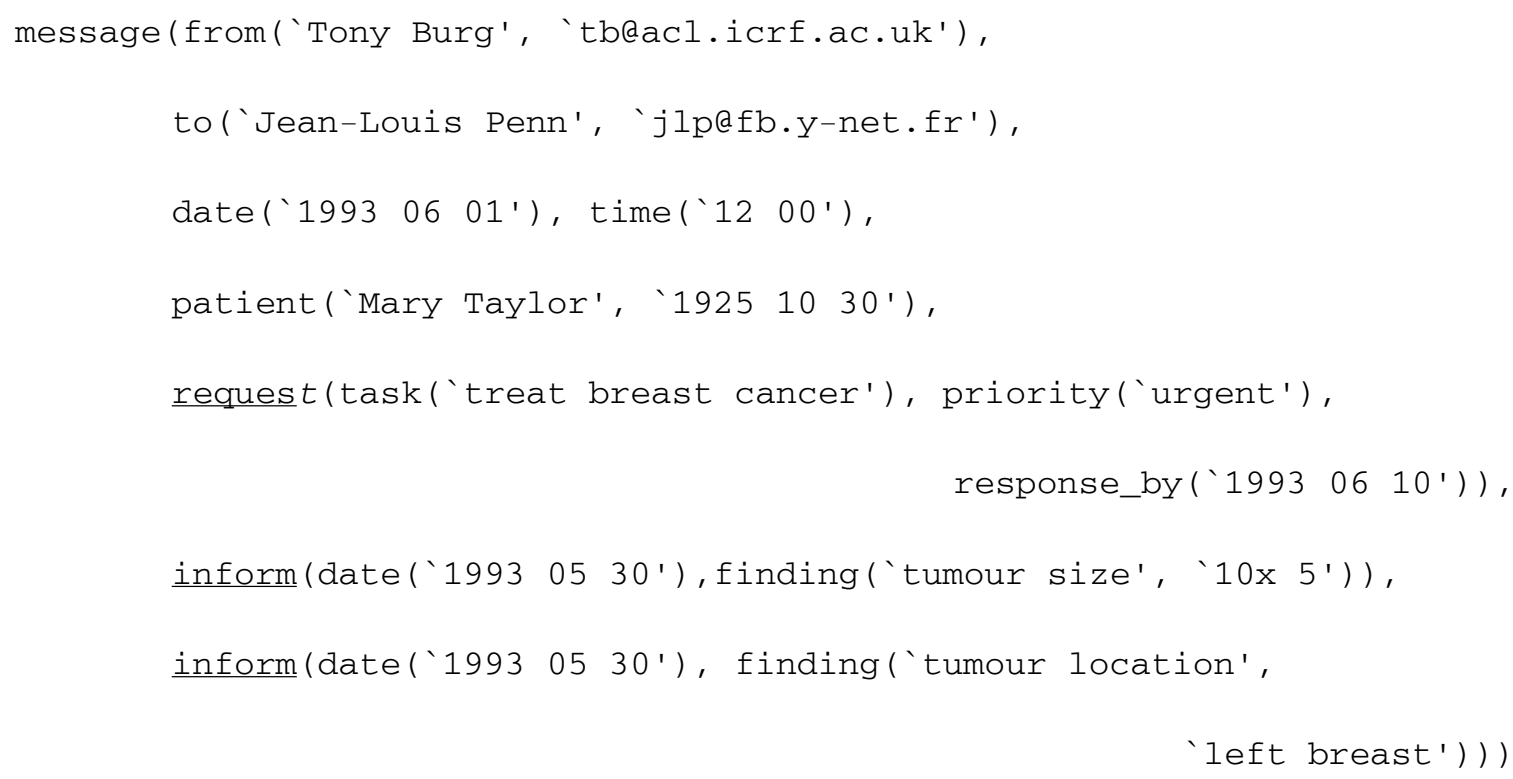

Jean-Louis Penn's communications manager converts this message into three primitives (i.e. request, inform and inform) and add them into its working memory. The arrival of these new primitives then triggers the control layer to apply the cooperation rules to evaluate task requests and generate commitments where appropriate. The result is that Jean-Louis Penn agrees to undertake the requested task and consequently an accept primitive is generated and then composed by the communications manager into a complete outward message to Tony Burg: 


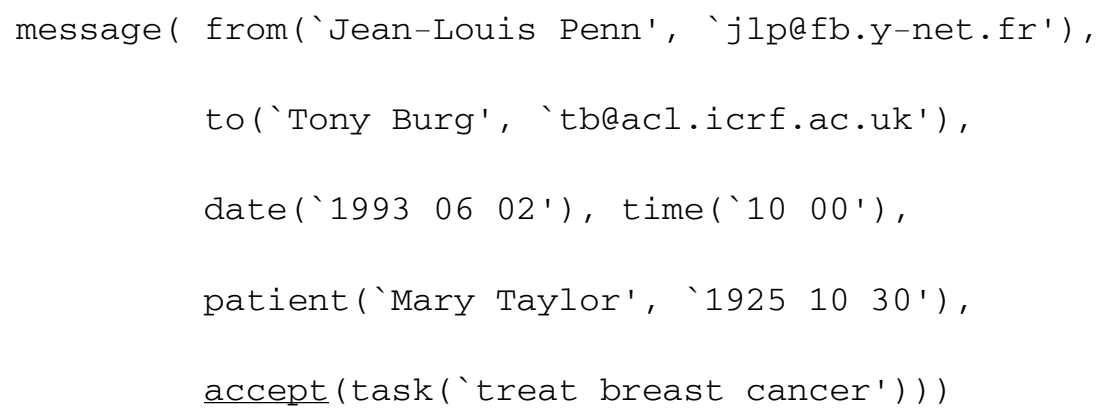

\subsection{Symbolic Decision Making}

The purpose of the decision rules is to choose among alternative options (e.g. potential diagnoses of a patient's illness and potential clinical protocols which could be used to treat the patient). As well as being used to decide which course of action to start, these rules may also be embedded as a decision point within the body of an action - eg whilst executing a particular clinical protocol there may be a crucial decision to be made which needs to make use of the decision making know-how contained in this rule group (see section 3.3 for an illustration of this point with respect to prescribing).

In this application domain, decision making is often complicated by the presence of incomplete or even conflicting information. For example, a drug may be very effective for eliminating a tumour, but the patient may be unwilling to tolerate its side effects. To facilitate decision making in such a context, a domain-independent decision procedure is abstracted and separated from domain-specific knowledge: the same set of decision rules can then be used to make decisions in varying medical domains (such as cancer, diabetes, and cardiology). Such a separation also permits formalisation of the decision knowledge.

The starting point of a decision making session is a goal, represented as a decision context, which is either given by the user or generated by the task management rules (section 3.3). By way of an example, the agent could have a goal of deciding which 
clinical protocol to select to treat a patient with breast cancer. Given this context, there are several distinct components of the decision procedure. The primary component activities are proposing candidate decision options, refining candidates, arguing the pros and cons of the options in view of the available evidence (argument generation), and aggregating the arguments to determine the preferred option (argument aggregation). For instance, the use of chemotherapy and radiotherapy may be proposed to treat the breast cancer of an old-aged patient (proposing). These options may then be refined to specific chemotherapy and radiotherapy treatments (refining). Arguments supporting the use of a particular chemotherapy treatment may include its effectiveness for removing the cancer, but there may also be arguments against its use (e.g. the level of toxicity associated with the drug may be too high for this particular patient due to her age). The pros and cons for each proposed option are finally combined to give the most preferred decision - e.g. the decision to use CT1 chemotherapy (argument aggregation).

This decision procedure is based on a simple but flexible method of reasoning under uncertainty for argument generation and aggregation, called argumentation (Krause et al., 1993), which avoids the necessity for precise quantification of uncertainty. Argumentation involves two simple ideas. First, one may know that some piece of information increases one's belief in a diagnosis, or preference for an action, though one may not be able to put a precise number on the change. Arguments for options can be constructed that are qualitatively labelled to indicate this change - for example, "confirm", "support", "weaken" or "exclude". Arguments of this sort are similar to Cohen's endorsements (Cohen, 1985), but in this work a more sophisticated set of aggregation functions are used to combine collections of arguments to yield a preference ordering on the decision options. This method is versatile, conceptually intuitive, relatively easy to implement, and 
simplifies some of the problems of knowledge acquisition and maintenance. The second idea is that the grounds of arguments for and against decisions are explicitly represented meaning they can serve a variety of functions including truth maintenance and explanation.

An example inference schema in the decision procedure specifies that if a decision candidate is proposed for a decision context and supported by a known clinical or nonclinical finding, then a supporting argument for the candidate is derived for the decision context:

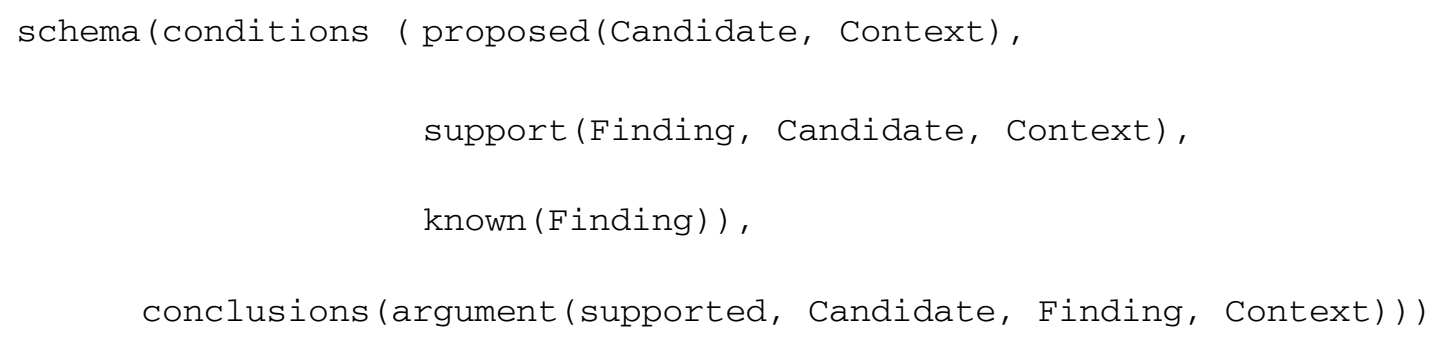

To take an example, suppose that the CT1 protocol has been proposed as a possible treatment protocol for breast cancer. Given the following patient data (patient is old) and domain knowledge (the $\mathrm{CT} 1$ protocol is known to be appropriate for treating breast cancer in elderly patients):

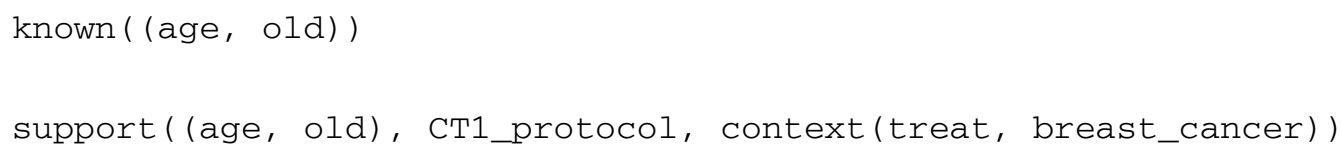

the following argument is derived using the above inference schema:

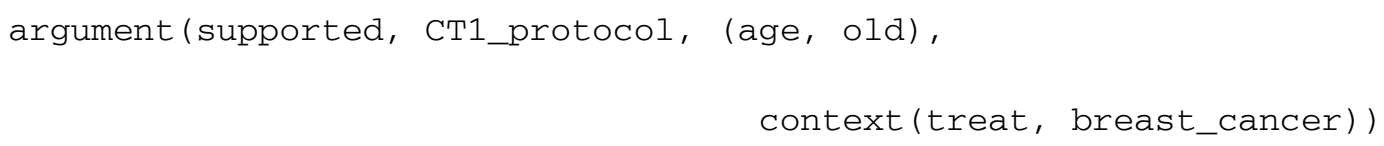


Further details of this approach to decision making are given elsewhere - for example, Fox and Krause (1992) describe it within a general context of qualitative reasoning and Huang et al. (1993) give a more formal, declarative specification of the decision procedure and discusses its application in medical decision making - and therefore will not be elaborated upon here. The emphasis in this paper is on the use of this generic decision knowledge alongside task management and cooperation knowledge in an integrated agent architecture for coordinated care. For example, the decision rules select an appropriate clinical protocol, which is then decomposed, allocated and monitored by the task management and cooperation rules.

\subsection{Task Management}

Once the decision procedure has selected a particular clinical protocol to achieve the agent's goal, the task manager component is responsible for its decomposition into subtasks, the allocation of subtasks to appropriate agents, and the management of task state transitions. Each of these activities is described in turn in the remainder of this section.

The structure of a generic clinical plan (e.g. CT1 for treating breast cancer) is determined by experts in authority, and is precisely defined in a clinical protocol (see figure 1). The task management rules decompose such a protocol into subtasks according to the predefined plan structure (as described in section 2). Subtasks at the bottom of the plan hierarchy may be primitive actions for humans or machines to perform (such as 'allocate bed' and 'administer drugs') or they may be decision tasks (such as choosing the right drug for a patient). In the latter case, the decision procedure is used to perform such a task, as explained in section 3.2. 
To facilitate task allocation, there are two roles associated with each (sub)task within the system - there is one agent who manages the execution of the task (i.e. ensures that it gets executed by somebody within the system and that the result of the execution is sent back to the originator) and one agent who is actually responsible for performing the task (the contractor). Task allocation is, therefore, the process by which the manager of a task finds the most appropriate contractor to perform it. The key structure in AADCare for making such decisions is that of accountability. Accountability is a static relationship which defines for what and to whom an agent is responsible. It is expressed by the following relation: accountable(Agent1,Agent2, TaskType) which means that Agent1 is accountable to Agent2 for performing tasks of type TaskType. For example, a hospital nurse may be accountable to one or more doctors for monitoring patient data such as temperature and blood pressure. The task manager component uses its accountability relations, together with the generic inference rule given below, to pick the most appropriate contractor for a given task. The underlined term "request" represents a primitive which is sent to the communications manager when this task management rule is fired (as described in section 3.1).

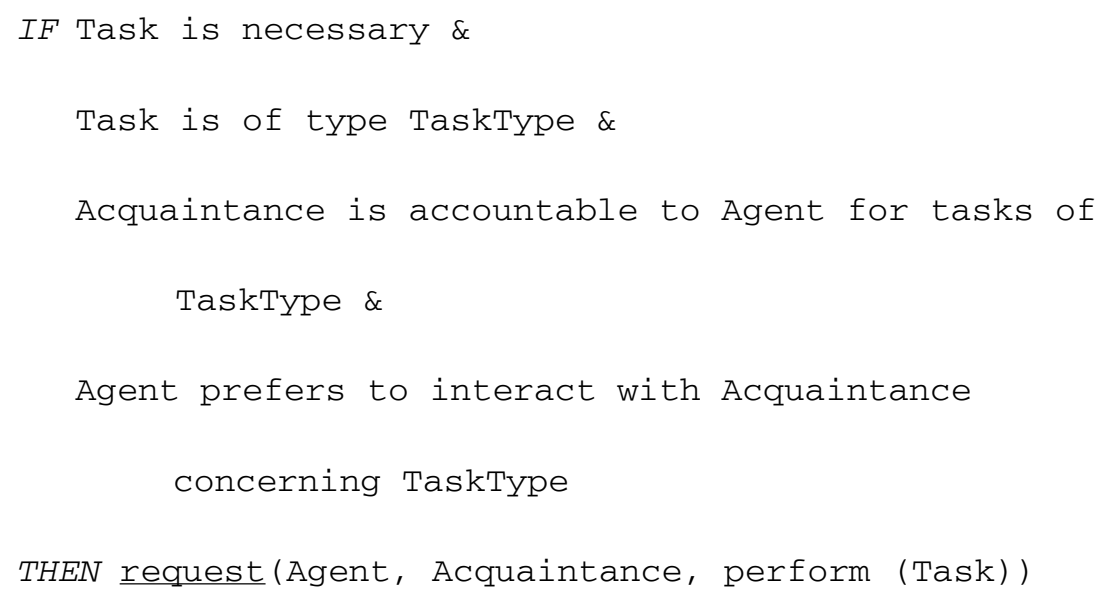


All tasks within AADCare have a state (either scheduled, cancelled, started, completed, or abandoned). The management of the transitions between these states needs to be carefully controlled by the agents because such transitions need to be documented in patients' care records: for example, when a task was scheduled, when it was started, when it was completed and when (why) it was abandoned. Transition management is complicated by the nested structure of the care plans. For example, the following two task management rules specify that when a composite task is cancelled, its started subtasks become abandoned and the subtasks that are scheduled but not yet started become cancelled:

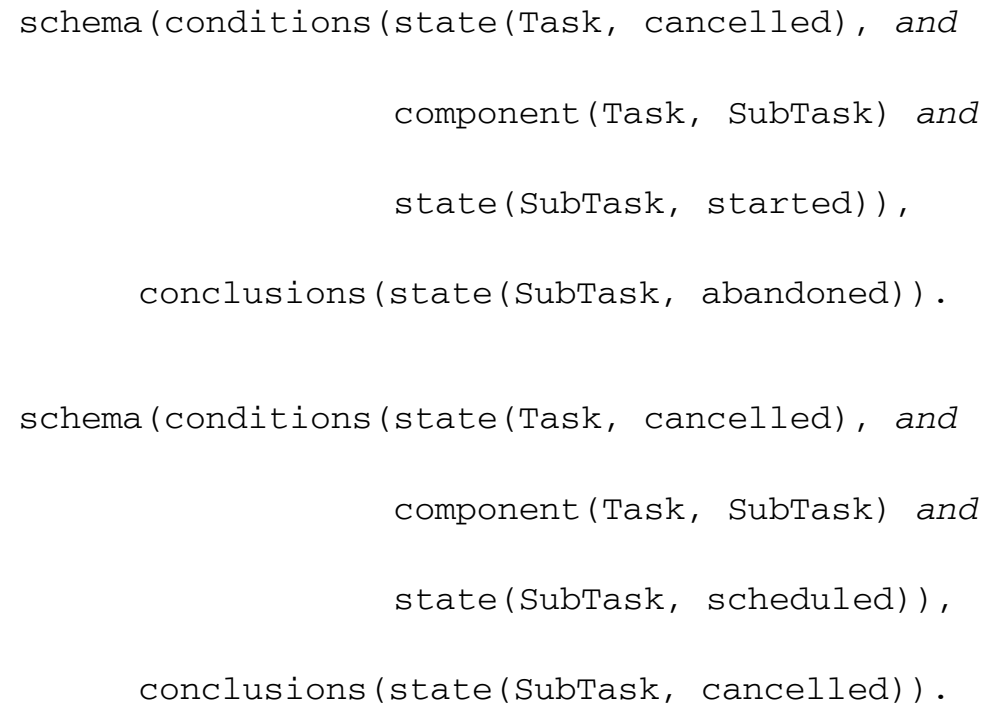

A distinction is made between the states of cancelled and abandoned because a corrective action is usually needed for an abandoned task (e.g. when the patient has to stop taking a certain drug which he has already been taking for a period) whereas such action is not normally necessary for a cancelled task.

\subsection{Managing Agent Cooperation}

In AADCare, the underlying mechanisms on which cooperative interactions are based are those of commitment (pledge to undertake a specified course of action) and convention 
(means of monitoring commitments in changing circumstances) (Jennings, 1993). The former means that if an agent agrees to undertake a task then it will endeavour to execute it at the appropriate time - this implies both that the agent is able to perform the task and that it has the necessary resources. Conventions are needed because commitments are not irrevocable: agents' circumstances may change between the making and the execution of their commitments, and agreed actions may turn out to be undesirable or even impossible to perform. Conventions, therefore, define the conditions under which an agent can drop its commitments and how to behave with respect to other agents in the cooperating group when such circumstances arise.

Given that cooperation is founded on commitments and conventions, two key issues need to be addressed: (i) what is involved in establishing a commitment? (section 3.4.1); and (ii) what type of convention is appropriate for monitoring commitments in the given care organisation? (section 3.4.2).

\subsubsection{Establishing commitments}

Accountability alone does not guarantee commitment: to commit to a specified task, an agent must also have the necessary resources (temporal and material) which are required to perform that task ${ }^{*}$. For example, a hospital specialist may be accountable to patients for in-hospital breast cancer treatment, but will not become committed to an actual treatment

\footnotetext{
* In addition, an agent may also have a local policy governing the acceptability of a requested task. For instance, a hospital may specify the following internal policy: a patient can only be admitted to the hospital if his/her GP is suitably registered with the hospital (so that the hospital can be paid more quickly). The capture and use of these policies remains a challenge to computer-assisted care and so, for the sake of simplicity, it is assumed here that availability of the appropriate resources is the only requirement for an agent to commit to a task.
} 
course on a specific patient until the time (temporal resource) and a bed (material resource) are available to perform the treatment. Although agents know what resources are available to themselves, they do not generally have information about the resources of their acquaintances. Therefore an agent may have to propose the same task to several acquaintances before an acceptable contractor, and hence commitment, can be found (made).

When an agent accepts a request it becomes committed to performing it (i.e. it takes on the role of contractor) and informs the manager that the task has been accepted using the following inference rule:

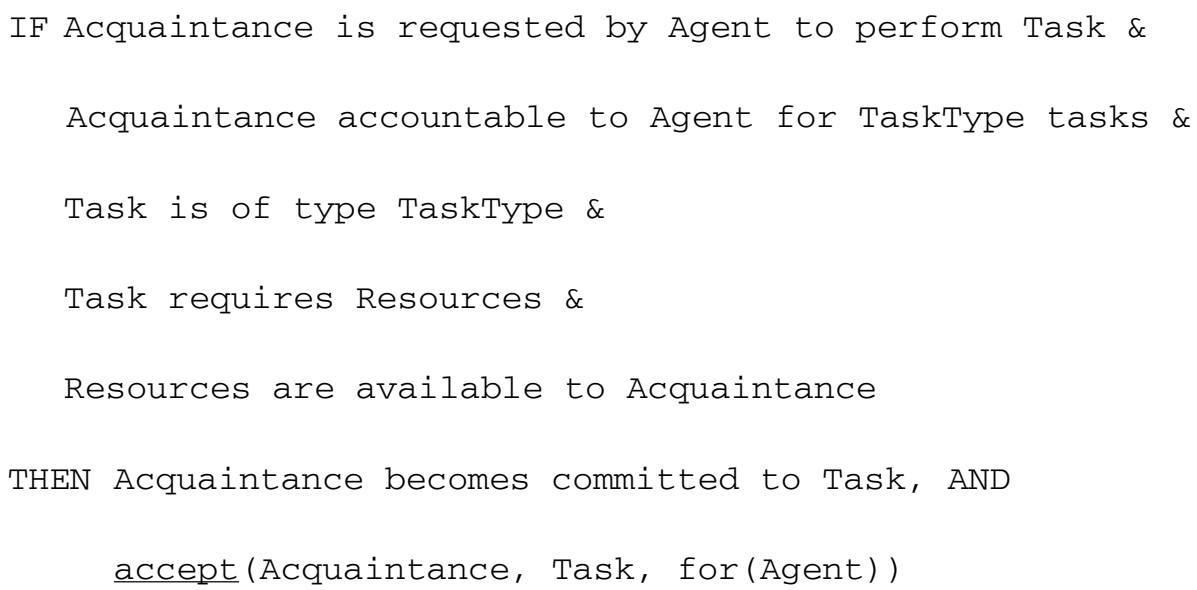

Commitment to the role of contractor also entails an additional responsibility - when the task has been completed the contractor must inform the manager about it and any results which have been generated - again this behaviour is encoded in a generic inference rule:

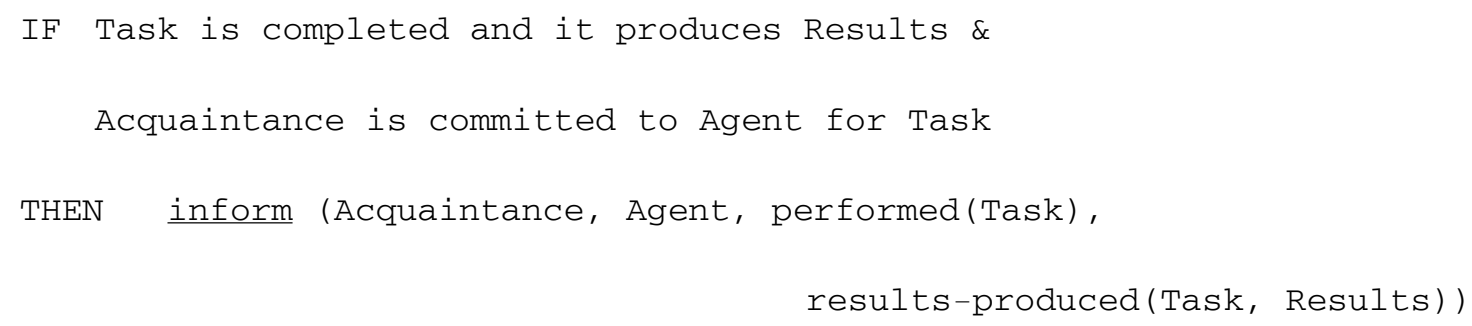


Note that in both cases, the underlined term represents primitives sent to the communication manager when the appropriate inference rules are triggered (as described in section 3.1).

\subsubsection{Adaptive management of commitment changes}

In most cases, when an agent commits itself to perform a task then that task will indeed be executed. However in certain well-defined circumstances it may be appropriate for an agent to renege upon its commitment. There may be an unforeseen lack of resources (e.g. unrelated emergencies may arise), the need for the task may cease to exist (e.g. because of the unexpected death of the patient), or it may no longer be feasible to execute a given task (e.g. a planned chemotherapy may have to be withdrawn because the patient has a high temperature resulting from the toxic effect of the drug). Having detailed the conditions under which commitments can be cancelled, the convention must also specify how to manage this change both locally and within the wider context of the cooperating group. The latter is important because it ensures that the cooperating care agents will behave coherently in the face of dynamic and unpredictable changes in the network (Jennings, 1995). Figure 3 details the convention embodied in the AADCare cooperation manager for the breast cancer treatment prototype.

\section{$<$ FIGURE 3>}

\section{Related Work}

In this section AADCare is briefly compared with some of the well-known architectures and systems in the agent literature.

GRATE (Jennings et al., 1992) is also a layered architecture that provides a generic 
cooperation module, situation assessment module, control module, and applicationspecific module. However the GRATE framework lacks an uncertainty management mechanism which is essential for medical decision making. Also GRATE's layers are functionally separated rather than logically separated - the additional benefit of this logical separation is that it provides a convenient basis for declarative specification and for logical verification and validation of the various layers of knowledge (eg in a formal language such as $\mathrm{ML}^{2}$ (van Harmelen, 1992)). The same two observations can be made of other, similar layered architectures such as INTERRAP (Muller et al., 1995) and TouringMachines (Ferguson, 1995).

Coordinator (Winograd and Flores, 1986) is a conversational system for coordinated action which is based on Searle's speech act theory (Searle, 1969). However, whilst the generation and monitoring of speech acts and commitments are centralised in Coordinator, AADCare distributes both of these functions (thus helping to reduce the communication bottleneck). Also the functionality of Coordinator is limited to coordination alone through the generation of speech acts and commitments, whereas AADCare accommodates additional functions such as a generic decision module for decision making under uncertainty.

AADCare also bears certain similarities to a standard blackboard architecture (Engelmore and Morgan, 1988). In both cases the working memory is changed through the application of functionally separated modules of inference rules. However, in addition to functional separation, AADCare also emphasises the logical layering of knowledge for reasons stated above and provides a set of generic knowledge modules for cooperation and decision making. 


\section{Conclusions}

Numerous techniques and systems have been developed in the medical informatics community for tackling isolated aspects of medical decision making. However, despite a well-documented need for supporting an integrated range of different functions (including uncertainty management, task management and coordination), there has been very little prior work which attempts to provide comprehensive procedures and integrated decision support for these different aspects of health care. AADCare therefore represents an important first step towards providing this integrated support. It gives a novel coupling of a decision making procedure and DAI techniques for task management, cooperation and communication. Such a coupling is essential if the full potential of automation is to be attained in the important real world domain of health care management.

A prototype AADCare system has been developed for the specific application of distributed management of cancer patients among general practices, hospitals, home care organisations and pharmacies. PROLOG is used for the representation of the domain- and inference- layer knowledge, and a production-rule language, implemented in PROLOG, is used for the data-driven control. A standard email system (Microsoft Mail) and server is used for message passing among the care agents. This system has been installed on a network of PCs running LPA-Prolog and MAPI (messaging applications interface written in C) under Microsoft-Windows 3.1 for Workgroups.

Preliminary evaluation of this prototype indicates that in real clinical application settings where exact probabilities and utilities are difficult to obtain the built-in symbolic decision procedure is more effective than conventional numerical methods (Walton and Randall, 1992). Also a senior oncologist manager and a senior cardiologist manager 
concluded that the cooperation strategy would provide useful guidance for clinicians jointly executing a care programme. Desirable extensions to the current work would be to interface the prototype system to existing patient information systems and electronic healthcare information networks. Once these interfaces are established, it is envisaged that AADCare technology will be used in operational settings to greatly improve the delivery of effective, efficient and globally coherent patient care programmes.

\section{Acknowledgments}

The first and third authors are grateful to the EC Advanced Informatics in Medicine (AIM) programme for funding the DILEMMA project in which this work was carried out. The role of the second author has been to assist in the cooperation and communication aspects of the work. 


\section{References}

Cohen, P. R. 1985. Reasoning about Uncertainty: An Artificial Intelligence Approach. Pitman, London.

Engelmore, R., and Morgan, T. 1988. Blackboard Systems. Addison-Wesley.

Ferguson, I. A. 1995. Integrated control and coordinated behaviour: a case for agent models in Intelligent Agents (eds. M. J. Wooldridge and N. R. Jennings), Lecture Notes in Artificial Intelligence, Volume 890, Springer Verlag.

Fox, J., Glowinski, A., Gordon, C., Hajnal, A., and O’Neil, M. 1990. Logic Engineering for Knowledge Engineering: Design and Implementation of the Oxford System of Medicine. Artificial Intelligence in Medicine 2, pp 323-339.

Fox, J., and Krause, P. 1992. Qualitative frameworks for decision support: lessons from medicine. Knowledge Engineering Review 7, pp 19-33.

Gordon, C., Herbert, S. I., Jackson-Smale, A., and Renaud-Salis, J. L. 1993. Care protocols and healthcare informatics. Proc. of Artificial Intelligence in Medicine Europe 93, Munich, Germany, pp 289-309.

Hickman, F. R., Killin, J. L., Land, L., Mulhall, T., Porter, D., and Taylor, R. M. 1989. Analysis for Knowledge Based Systems: a Practical Guide to the KADS Methodology. Ellis Horwood, Chichester.

Huang J., Fox J., Gordon C., and Jackson-Smale A. 1993. Symbolic decision support in Medical Care. Artificial Intelligence in Medicine 5, pp 415-430.

Huang, J., Jennings, N. R., and Fox, J. 1994. Cooperation in Distributed Medical Care. 
Proc. of Second Int. Conf. on Cooperative Information Systems, Toronto, Canada, pp 255263.

Huang, J., Jennings, N. R., and Fox, J. 1995. An Agent Architecture for Distributed Medical Care. in Intelligent Agents (eds. M. J. Wooldridge and N. R. Jennings) Lecture Notes in Artificial Intelligence, Volume 890, Springer Verlag, pp 219-232.

Jennings N. R. 1993. Commitments and conventions: the foundation of coordination in multi-agent systems. Knowledge Engineering Review 8, pp 223-250.

Jennings, N. R. 1995. Controlling Cooperative Problem Solving in Industria Multi-Agent Systems using Joint Intentions. Artificial Intelligence 74 (2).

Jennings N. R., Mamdani E. H., Laresgoiti I., Perez J., and Corera J. 1992. GRATE: a general framework for cooperative problem solving. Intelligent Systems Engineering 1 (2) pp 102-114.

Krause, P., Amble, S., and Fox, J. 1993. The Development of a Logic of Argumentation. in: Advanced Methods in Artificial Intelligence, Lecture Notes in Computer Science Series, Springer-Verlag, Berlin

Lux A, de Greef P, Bomarius F and Steiner D. 1993. A generic framework for human computer cooperation. Proc. of Int. Conf. on Intelligent and Cooperative Information Systems (ICICIS-93), Rotterdam, the Netherlands, pp. 89-97.

Muller, J. P., Pischel, M., and Thiel, M. 1995. A pragmatic approach to modelling autonomous interacting systems: a preliminary report. in Intelligent Agents (eds. M. J. Wooldridge and N. R. Jennings), Lecture Notes in Artificial Intelligence, Volume 890, Springer Verlag. 
Pritchard, P. 1992. The role of computers in referral. in Referrals to Medical Outpatients (eds. Hopkins, A. \& Wallace, P.), Royal Colleges of Physicians and General Practitioners, pp 79-89

Reeves, P., Rickards, T., and Carniel, B. 1993. Requirements for Shared Care Decision Support in Cardiology. Technical Report, Royal Brompton National Heart and Lung Hospital, London, England.

Renaud-Salis, J. L., Lagouarde, P., Gordon, C., and Thomson, R. 1992. Requirements for Decision Support in Cancer Shared Care. Technical Report, Fondation Bergonie, Bordeaux, France.

Searle, J. R. 1969. Speech Acts: an Essay in the Philosophy of Language Cambridge University Press.

Shoham, Y. 1993. Agent-oriented programming. Artificial Intelligence. 60 (1) pp 51-92.

Smith R. G. 1980. The contract net protocol: high-level communication and control in a distributed problem solver. IEEE Transaction on Computers, C-29 (12), pp 1104-1113

van Harmelen, F. 1992. ML $\mathrm{ML}^{2}$ a Formal Language for KADS Models of Expertise. Knowledge Acquisition 4 (1).

Walton, R., and Randall, A. 1992. Clinical Decision Analysis. British Medical Journal $301, \mathrm{p} 301$.

Winograd, T., and Flores, F. 1986. Understanding Computers and Cognition: a New Foundation for Design. Ablex Publishing, Norwood.

Wooldridge, M. J., and Jennings, N. R. 1995. Agent Theories, Architectures and 
Languages: A Survey. in Intelligent Agents (eds. M. J. Wooldridge and N. R. Jennings), Lecture Notes in Artificial Intelligence, Volume 890, Springer Verlag, pp 1-32. 


\begin{tabular}{|c|c|c|}
\hline Type & Content & Effect on receiver \\
\hline request & $\begin{array}{l}\text { task; } \\
\text { [provisional schedule]; } \\
\text { priority: urgent or not; } \\
\text { response_by date }\end{array}$ & $\begin{array}{l}\text { ReceiveAgent evaluates whether to accept the } \\
\text { request, and informs SendAgent of decision. If } \\
\text { recipient decides to accept the request, it } \\
\text { becomes committed to the task. }\end{array}$ \\
\hline accept & $\begin{array}{l}\text { task; } \\
\text { [accepted schedule] }\end{array}$ & $\begin{array}{l}\text { ReceiveAgent knows SendAgent is committed to } \\
\text { the request and that SendAgent will inform it of } \\
\text { the outcome of executing the task. SendAgent } \\
\text { becomes the contractor for the task and } \\
\text { ReceiveAgent the manager. }\end{array}$ \\
\hline reject & $\begin{array}{l}\text { task; } \\
\text { [provisional schedule] }\end{array}$ & $\begin{array}{l}\text { ReceiveAgent has to request someone else to } \\
\text { perform the task on the provisional schedule }\end{array}$ \\
\hline alter & $\begin{array}{l}\text { task; } \\
\text { provisional schedule; } \\
\text { acceptable schedule }\end{array}$ & $\begin{array}{l}\text { ReceiveAgent to evaluate the acceptable sched- } \\
\text { ule and decide whether to replace the provisional } \\
\text { schedule with the acceptable schedule. If so, it } \\
\text { sends SendAgent a new request. Otherwise, } \\
\text { ReceiveAgent has to send the original request to } \\
\text { someone else }\end{array}$ \\
\hline propose & $\begin{array}{l}\text { task; } \\
\text { [proposed schedule] }\end{array}$ & ReceiveAgent may or may not adopt the proposal \\
\hline inform & $\begin{array}{l}\text { any information: data, domain } \\
\text { knowledge or partial plans }\end{array}$ & $\begin{array}{l}\text { ReceiveAgent may use the information for local } \\
\text { problem solving }\end{array}$ \\
\hline query & $\begin{array}{l}\text { a question: what, how, } \\
\text { whether, and so on }\end{array}$ & $\begin{array}{l}\text { ReceiveAgent must answer the query, possibly } \\
\text { involving extensive local problem solving (e.g. } \\
\text { diagnosis and investigation). A reply may be of } \\
\text { type 'propose'. It may also be 'inform', possibly } \\
\text { giving the answer 'unknown' to the query }\end{array}$ \\
\hline cancel & $\begin{array}{l}\text { any message of the above } \\
\text { types }\end{array}$ & ReceiveAgent should ignore the earlier message \\
\hline acknowledge & $\begin{array}{l}\text { any message of the above } \\
\text { types. All messages need to } \\
\text { be acknowledged except } \\
\text { acknowledgement messages } \\
\text { themselves }\end{array}$ & $\begin{array}{l}\text { ReceiveAgent is aware of the successful trans- } \\
\text { mission of the message }\end{array}$ \\
\hline
\end{tabular}

Table 1: Communication primitives 


\section{Figure Captions}

Figure 1: Part of CT1 protocol for treating breast cancer

Figure 2: AADCare Agent architecture

Figure 3: Convention for Adapting Commitments in AADCare 


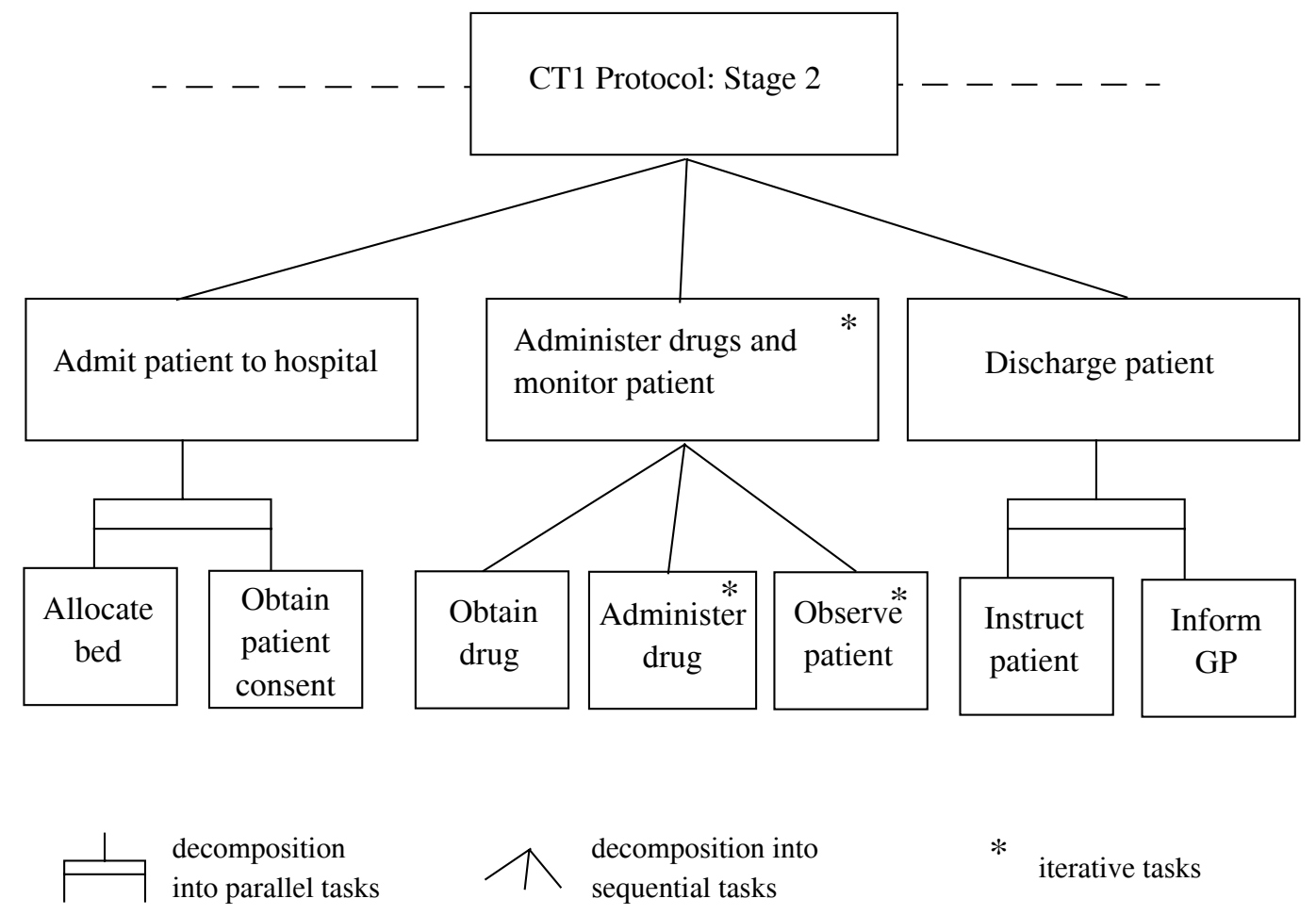

Figure 1: Part of CT1 protocol for treating breast cancer 


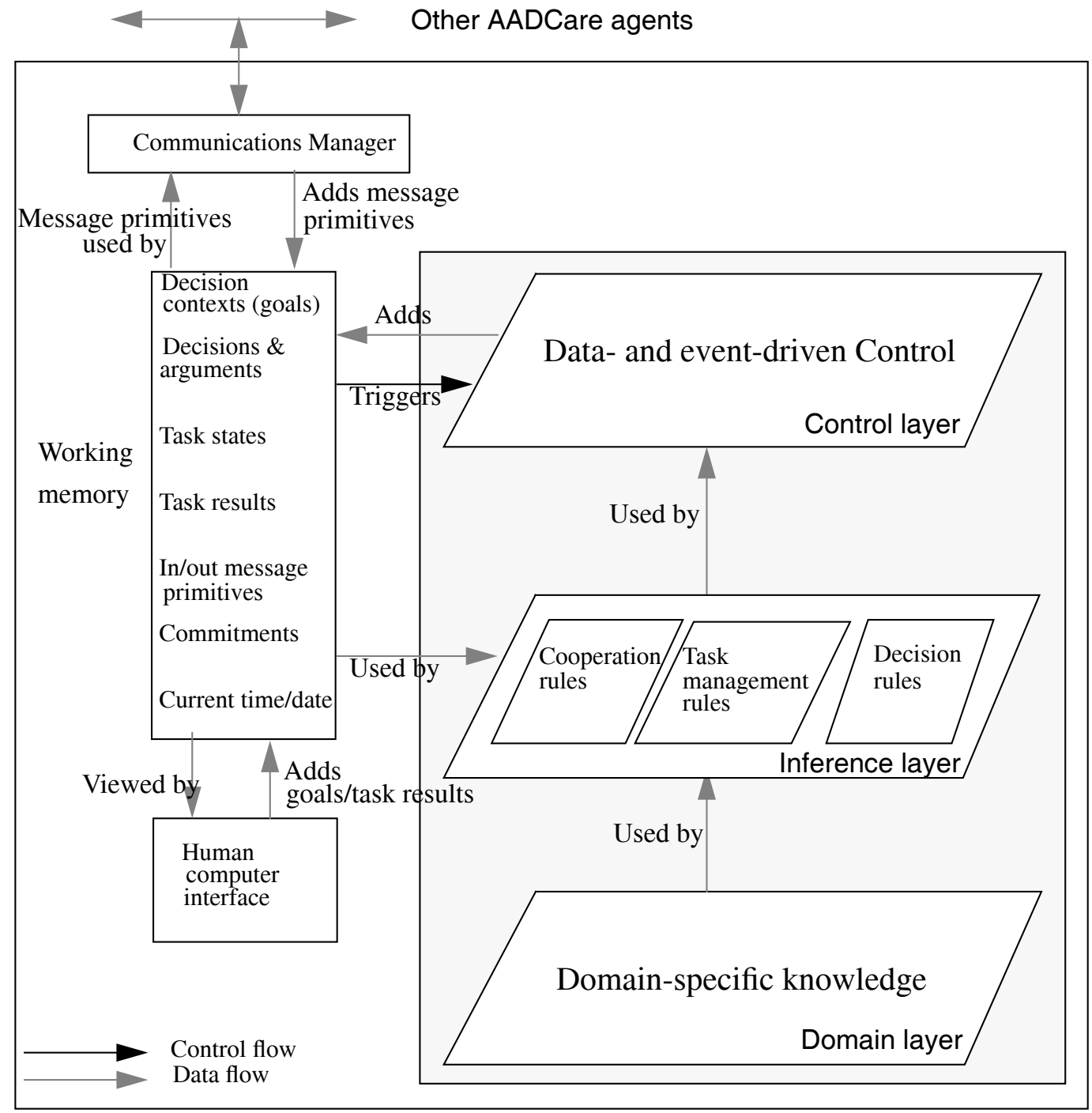

Figure 2: AADCare Agent architecture 


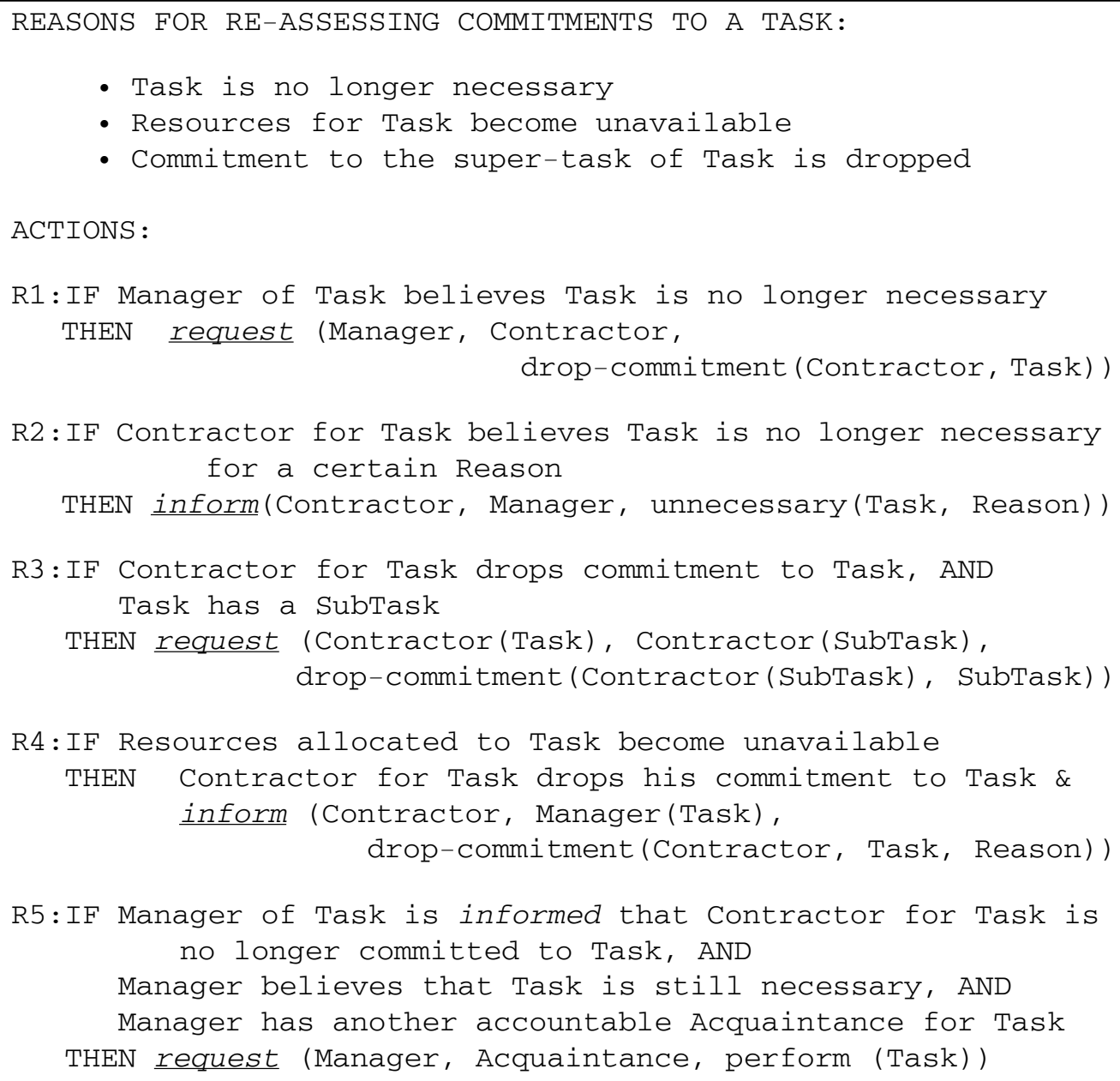

Figure 3: Convention for Adapting Commitments in AADCare 